\title{
Possible paracrine growth of adenocarcinoma of the stomach induced by granulocyte colony stimulating factor produced by squamous cell carcinoma of the oesophagus
}

\author{
E Ichiishi, T Yoshikawa, T Kogawa, N Yoshida, M Kondo
}

\begin{abstract}
Synchronous cancers of the oesophagus and stomach diagnosed in a patient showing pronounced leucocytosis were examined for production of granulocyte colony stimulating factor (G-CSF) and expression of G-CSF receptor. Whereas enzyme immunoassay of tissue extracts showed that the oesophageal carcinoma produced G-CSF, the gastric cancer did not. However, the gastric tumour showed G-CSF receptor expression on immunohistochemical examination of sections. These findings suggest that the oesophageal cancer promoted gastric cancer growth by paracrine mechanisms involving G-CSF. (Gut 2000;46:432-434)
\end{abstract}

Keywords: cancer; gastric; oesophageal; stomach; granulocyte colony stimulating factor; receptor

A striking leucocytosis without evidence of infection is known to occur occasionally in patients with non-haematological neoplasms that elaborate colony stimulating factors. Production of granulocyte colony stimulating factor (G-CSF) has been reported in lung cancers as well as carcinomas of the gall bladder, liver, stomach, pancreas, and thyroid. This report describes a patient with synchronous oesophageal and gastric carcinomas accompanied by pronounced leucocytosis. Whereas only the oesophageal carcinoma produced G-CSF, the cells of the gastric carcinoma strongly expressed G-CSF receptor protein. As G-CSF receptor expression has been implicated in the progression of malignant behaviour in nonhaematopoietic cancers, ${ }^{1}$ the findings in our case suggest the possibility of paracrine stimulation.

\section{Case report}

A 66 year old Japanese man was admitted to hospital in March 1995, the main complaint being general malaise. Laboratory tests showed pronounced leucocytosis (33 900/ $\mu$ l with $55 \%$ band forms, $42 \%$ segmented forms, and $3.0 \%$ lymphocytes). Serum concentrations of carbohydrate antigen 19-9 and squamous cell carcinoma antigen were elevated to $98 \mathrm{U} / \mathrm{ml}$ and
Table 1 Concentrations of granulocyte colony stimulating factor (G-CSF) in extracts of tumour tissue and normal tissue measured by enzyme immunoassay using abti-recombinant G-CSF antibody

\begin{tabular}{lll}
\hline & \multicolumn{2}{c}{$G$-CSF concentration $(p g / m l)$} \\
\cline { 2 - 3 } & Oesophagus & Stomach \\
\hline Tumour tissue & 180 & 15 \\
Normal tissue & 2 & 35 \\
\hline
\end{tabular}

$41.1 \mathrm{ng} / \mathrm{ml}$ respectively, while the serum carcinoembryonic antigen concentration was normal. Gastrointestinal endoscopy disclosed coexisting oesophageal and gastric carcinomas. An oesophageal tumour $7 \mathrm{~cm}$ in length extended nearly to the cardia. In the gastric antrum, an intestinal-type cancer was found. A bone marrow aspirate was hypercellular with almost normal percentages of various precursor cells. Cytogenetic examination did not detect a Ph1 chromosome. On 23 May, peripheral leucocyte count peaked at $110850 / \mu 1$. Progressive cachexia developed, and the patient died two months after admission. Only supportive care was given. Autopsy was performed one hour after death.

\section{Assay of tumour extracts for G-CSF}

Extracts from oesophageal and gastric tissues were prepared by previously described methods. ${ }^{2}$ Levels of G-CSF in extracts from the tumours and from nearby normal tissues were measured by an enzyme immunoassay using an antibody to recombinant G-CSF (rG-CSF). G-CSF levels in the extract from oesophageal carcinoma $(180 \mathrm{pg} / \mathrm{ml})$ were higher than in those from any other tissues including the gastric carcinoma $(2-35 \mathrm{pg} / \mathrm{ml}$; table 1). These results strongly suggest that G-CSF was produced by the oesophageal carcinoma.

\section{Histological findings}

Histological examination showed the oesophageal carcinoma to consist of moderately to poorly differentiated squamous cells. Broad

Abbreviations used in this paper: G-CSF, granulocyte colony stimulating factor; rG-CSF, recombinant G-CSF. 

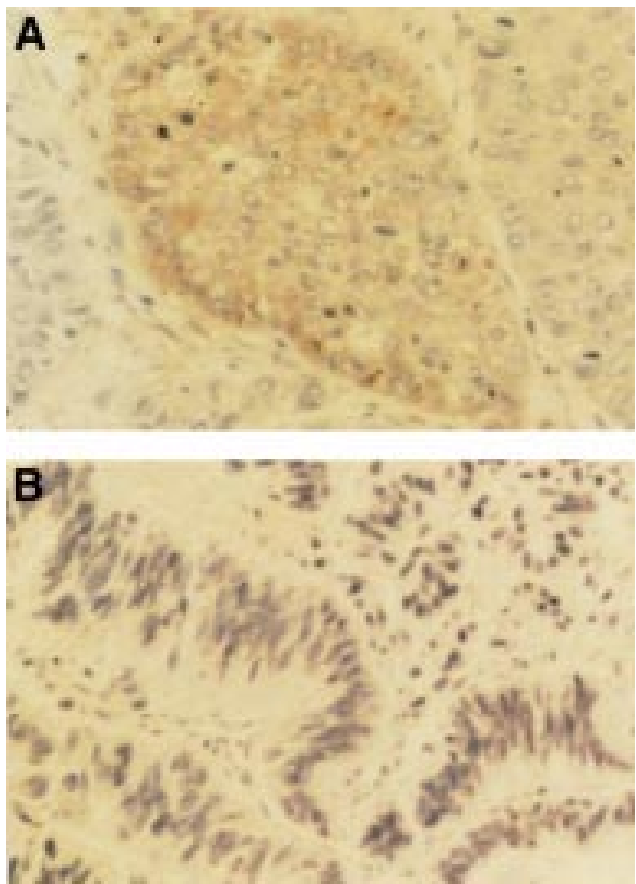

Figure 1 The tumour cells of the oesophageal carcinoma showed staining for monoclonal antibody against granulocyte colony stimulating factor $(G-C S F ; 4 A 6)$ in the cytoplasm $(A)$, but those of the stomach did not $(B)$.

areas of diffuse neutrophilic infiltration were seen. The gastric carcinoma was a well differentiated adenocarcinoma of the papillotubular type.

Immunohistochemical staining was performed in paraffin wax embedded tissue sections using a streptavidin-biotin labelling method. A monoclonal antibody against G-CSF (4A6) and a polyclonal antibody against G-CSF receptor (C-20) were used. Cells of the oesophageal carcinoma showed staining for G-CSF in the cytoplasm, but those of the gastric tumour did not (fig 1). Much stronger expression of the G-CSF receptor was seen on surfaces of cancer cells in the gastric tumour than on the cells of the oesophageal tumour (fig 2).

\section{Discussion}

G-CSF production has been found in cancers of the lung, ${ }^{2}$ gall bladder, ${ }^{3}$ liver, ${ }^{4}$ stomach, ${ }^{5}$ pancreas, ${ }^{6}$ and thyroid. ${ }^{7}$ An oesophageal carcinosarcoma elaborating G-CSF was reported by Ota et $a l,{ }^{8}$ who found production by both epithelial and mesenchymal tumour components. Only one of our patient's coexisting cancers produced G-CSF.

G-CSF, a haematopoietic cytokine, regulates proliferation and differentiation of granulocytic progenitor cells and functionally activates mature neutrophils. It also affects nonhaematopoietic tumour cells by binding to G-CSF specific receptors on their surfaces. G-CSF receptor expression by cancer cells may play a crucial role in mediating increasingly malignant behaviour of non-haematopoietic cancer cells, ${ }^{1}$ and autocrine growth promotion of malignant tumour cells by G-CSF has been suggested in human transitional carcinoma of the bladder. ${ }^{9}$ In our case, G-CSF production
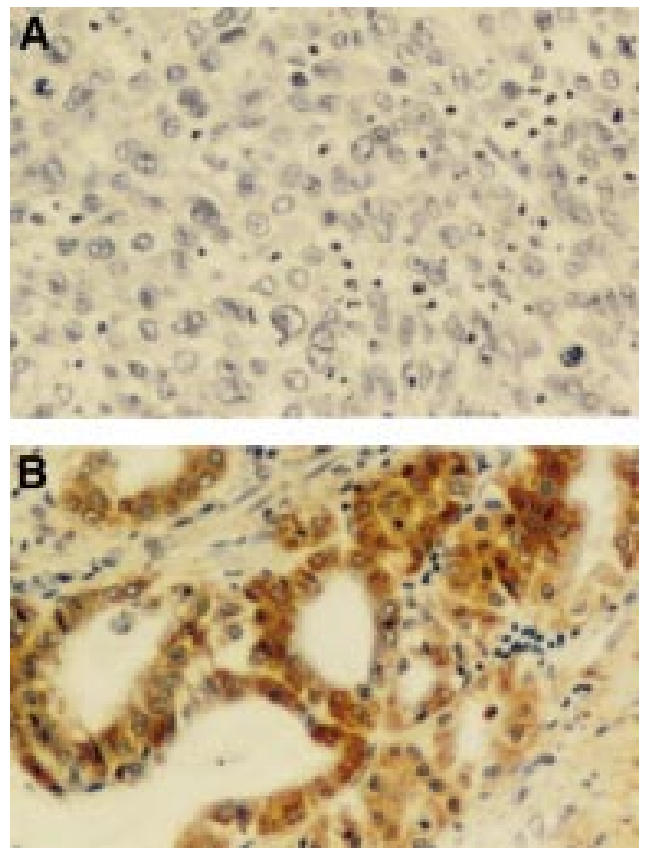

Figure 2 The tumour cells of the oesophageal carcinoma showed weak staining for polyclonal antibody against granulocyte colony stimulating factor $(G-C S F)$ receptor $(C-20)(A)$ compared with excessive expression on those of the stomach $(B)$.

by the oesophageal tumour and receptor expression by the gastric tumour may represent a paracrine regulation pattern.

In neutrophils, G-CSF receptors have two intracellular functional domains, one being essential for proliferation and the other needed for differentiation. These signals activate JAK1 tyrosine kinase families, such as interleukin-6, and tyrosine phosphorylation of the DNA binding protein STAT $3 .{ }^{10}$ When a G-CSF receptor expression plasmid was introduced into mouse myeloid precursor FDC-P1 cells, exogenous G-CSF stimulated proliferation of the transformants. ${ }^{11}$ G-CSF also induces human endothelial cells to migrate and proliferate through a G-CSF receptor. ${ }^{12} \mathrm{G}-\mathrm{CSF}$ has been reported to increase the invasive potential of head and neck carcinoma cells, and several intracellular events initiated by the binding of G-CSF to tumour cells are being explored. ${ }^{13}$ In vitro treatment of several head and neck carcinoma cell lines (IMC-2, IMC-3, KB, Ca9-22, SCCKN, and SCCTF) with rG-CSF has been found to augment their invasive potential considerably in a dose dependent and time dependent manner. Of these cancer cell lines, IMC-2, IMC-3, KB, and Ca9-22 produced little G-CSF, whereas large amounts of G-CSF were produced by SCCKN and SCCTF. AntiG-CSF antibody decreased invasiveness in the presence of rG-CSF to the level present in cancer cell lines not exposed to rG-CSF. Immunocytochemical staining and western blotting with an anti-G-CSF receptor monoclonal antibody disclosed expression of G-CSF receptor in head and neck cancer cell lines that had shown enhancement of invasive activity by rG-CSF. IMC-2 cells, which showed the highest invasiveness of the cell lines tested, showed augmentation of G-CSF receptor expression 
on stimulation with rG-CSF. Furthermore, stimulation of IMC-2 cells with rG-CSF induced rapid activation of tyrosine phosphorylated JAK1, suggesting that the G-CSF signal may be transduced into the cells through the G-CSF receptor. The gelatinolytic activity of IMC-2 cells was also enhanced by stimulation with rG-CSF, but this enhanced invasiveness was inhibited by the addition of tissue inhibitors of metalloproteinases. These results suggest that exogenous rG-CSF may increase the risk of metastasis or local recurrence in patients with G-CSF receptor positive head and neck squamous cell carcinoma as a result of enhanced invasiveness.

We hypothesised that G-CSF signals from our patient's oesophageal cancer cells may have contributed to the proliferative capacity of the gastric cancer cells, which showed aberrant expression of the G-CSF receptor. A human gastric cancer cell line, HuGC-OOHIRA, has previously shown increased proliferation caused by G-CSF, while an antibody raised against rG-CSF suppressed propagation of the HuGC-OOHIRA cell line. ${ }^{14}$ Therefore an autocrine growth promoting loop involving $\mathrm{G}-\mathrm{CSF}$ is operating in these cells.

As a control for our study, we immunostained six human gastric adenocarcinomas unassociated with leucocytosis for G-CSF as well as its receptor: none showed staining for G-CSF, but one showed staining for G-CSF receptor antibody (data not shown), consistent with only occasional G-CSF expression in solid tumours, in contrast with a high prevalence of expression in haematopoietic cancer cells. ${ }^{15}$ However, immunohistochemical staining does not confirm the presence of a functioning receptor, which requires binding assays for bioassays.

In recent years, the use of multimodel treatments involving the administration of G-CSF has increased dramatically, not only for malignant diseases but also for other intractable conditions such as chronic granulomatous disease. ${ }^{16}$ Using an in situ reverse transcription polymerase chain reaction, Tachibana and colleagues $^{17}$ investigated G-CSF receptor expression by tumour cells in human transitional cell carcinoma of the bladder. These authors found positive G-CSF receptor mRNA signals in six of 26 specimens. Because G-CSF receptor expression is often observed in bladder cancers, these authors cautioned that use of G-CSF to treat bladder cancer requires very careful patient selection.

The treatment of patients with cancers that are able to produce stimulating factors requires special care when it includes the administration of G-CSF or interleukin-3 for bone marrow recovery after chemotherapy or radiation of solid tumours. Repeated thorough evaluation for early detection of secondary cancers that may have been stimulated to grow by G-CSF mediated signals is essential. ${ }^{18} 19$

We gratefully acknowledge Dr Junichiro Fujimoto of the Department of Pathology, National Children's Medical Research Centre, for providing the G-CSF antibody (4A6), and $\mathrm{Mr}$ Daisuke Hashimoto of the Department of Laboratory Medicine, Social Insurance Kyoto Hospital, for skilful immunohistochemical assistance.

1 Uzumaki $\mathrm{H}$, Okabe $\mathrm{T}$, Sasaki $\mathrm{N}$, et al. Identification and characterization of receptors for granulocyte colonytimulating factor on human placenta and trophoblastic cells. Proc Natl Acad Sci USA 1989;86:9323-6.

2 Kimura N, Niho Y, Yanase T. A high level of colonystimulating activity in a lung cancer patient with extensive eukocytosis, and the establishment of a CSA producing cell line. Scand $\mathcal{F}$ Hematol 1982;28:417-24.

3 Egami H, Sakamoto K, Yoshimura R. Establishment of a cell line of gallgladder carcinoma producing human colony stimulating factor. Ipn $\mathcal{F}$ Cancer Res 1986;77:168-76.

4 Gabrilove J, Welte K, Lu L, et al. Constitutive production of leukemia differentiation, colony-stimulating, erythroid burst-promoting, and pluripoietic factors by a human hepatoma cell line. Blood 1985;66:407-15.

5 Kitanuma H, Kodama F, Odagiri S, et al. Granulocytosis associated with malignant neoplasms. Hum Pathol 1989;20: $878-85$

$6 \mathrm{Wu} \mathrm{M}$, Cini JK, Yunis AA. Purification of a colonystimulating factor from cultured pancreatic carcinoma cells. F Biol Chem 1979;254:6626-8.

7 Okabe T, Nomura H, Ohsawa N. Establishment and characterization of a human colony-stimulating factorproducing cell line from a squamous cell carcinoma of the thyroid gland. F Natl Cancer Inst 1982;69:1235-43.

8 Ota S, et al. A case of carcinosarcoma of the oesophagus where both epithelial and mesenchymal components produced G-CSF, hence suggesting a clonal origin. Cancer 1998;82:2102-11.

9 Tachibana M, Miyakawa A, Tazaki H, et al. Autocrine growth of transitional cell carcinoma of the bladder induced by granulocyte-colony stimulating factor. Cancer Res 1995;55:3438-43.

10 Tian S, Lamb P, Seidel H, et al. Rapid activation of the STAT3 transcription factor by granulocyte colonySTAT3 transcription factor by gran
stimulating factor. Blood $1994 ; 84: 1760-4$.

11 Fukunaga R, Ishizaka-Ikeda E, Nagata S. Growth and differentiation signals mediated by different regions in the cytoplasmic domain of granulocyte colony-stimulating faccytoplasmic domain of granulocyte col
tor receptor. Cell 1993;74:1079-87.

12 Bussolino F, Wang JM, Defilippi P, et al. Granulocyte and granulocyte-macrophage-colony stimulating factors induce human endothelial cells to migrate and proliferate. Nature 1989;337:471-3.

13 Noda I, Fujieda S, Ohtsubo T, et al. Granulocyte-colonystimulating factor enhances invasive potential of human head-and-neck-carcinoma cell lines. Int $\mathcal{F}$ Cancer 1999;80: $78-84$.

14 Baba $M$, Hasegawa $H$, Nakayabu $M$, et al. Establishment and characteristics of a gastric cancer cell line (HuGCOOHIRA) producing high levels of G-CSF, GM-CSF and IL-6: the presence of autocrine growth control by G-CSF. Am $\mathcal{f}$ Hematol 1995;49:207-15.

15 Kuwaki T, Hosoi T, Hanazono Y, et al. Distribution of Kuwaki T, Hosoi T, Hanazono Y, et al. Distribution of
human granulocyte colony-stimulating factor receptors. human granulocyte colony-stim 7 Cancer Res 1990;81:560-3.

16 Myrup B, Valerius NH, Mortensen PB. Treatment of enteritis in chronic granulomatous disease with granuloenteritis in chronic granulomatous disease with gran
cyte colony stimulating factor. Gut 1998;42:127-30.

17 Tachibana M, Miyakawa A, Uchida A, et al. Granulocyte colony-stimulating factor receptor expression on human Br F Cancer 1997;75:1489-96.

18 Avalos BR, Gasson JC, Hedvat C, et al. Human granulocyte colony-stimulating factor: biological activities and receptor characterization on hematopoietic cells and small cell lung cancer lines. Blood 1990;75:851-7.

19 Wolfgang EB, Riedl SD, Steinhauser G, et al. Various human hematopoietic growth factors stimulate clonal growth of nonhematopoietic tumor cells. Blood 1989;73:80-3. 